\title{
EAES Recommendations for Recovery Plan in Minimally Invasive Surgery Amid COVID-19 Pandemic
}

\author{
Alberto Arezzo ${ }^{1} \oplus \cdot$ Nader Francis $^{2,3} \cdot$ Yoav Mintz ${ }^{4} \cdot$ Michel Adamina $^{5,6} \cdot$ Stavros A. Antoniou $^{7,8} \cdot$ Nicole Bouvy $^{9}$. \\ Catalin Copaescu ${ }^{10} \cdot$ Nicolò de Manzini $^{11}$. Nicola Di Lorenzo ${ }^{12}$. Salvador Morales-Conde ${ }^{13}$. Beat P. Müller-Stich ${ }^{14}$. \\ Felix Nickel $^{14}$. Dorin Popa ${ }^{15}$. Diana Tait ${ }^{16}$. Cenydd Thomas ${ }^{17}$. Susan Nimmo ${ }^{18}$. Dimitrios Paraskevis ${ }^{19}$. \\ Andrea Pietrabissa ${ }^{20}$. The EAES Group of Experts for Recovery Amid COVID-19 Pandemic
}

Received: 26 September 2020 / Accepted: 22 October 2020 / Published online: 10 November 2020

(c) The Author(s) 2020

\begin{abstract}
Background COVID-19 pandemic presented an unexpected challenge for the surgical community in general and Minimally Invasive Surgery (MIS) specialists in particular. This document aims to summarize recent evidence and experts' opinion and formulate recommendations to guide the surgical community on how to best organize the recovery plan for surgical activity across different sub-specialities after the COVID-19 pandemic.

Methods Recommendations were developed through a Delphi process for establishment of expert consensus. Domain topics were formulated and subsequently subdivided into questions pertinent to different surgical specialities following the COVID-19 crisis. Sixty-five experts from 24 countries, representing the entire EAES board, were invited. Fifty clinicians and six engineers accepted the invitation and drafted statements based on specific key questions. Anonymous voting on the statements was performed until consensus was achieved, defined by at least $70 \%$ agreement.

Results A total of 92 consensus statements were formulated with regard to safe resumption of surgery across eight domains, addressing general surgery, upper GI, lower GI, bariatrics, endocrine, HPB, abdominal wall and technology/research. The statements addressed elective and emergency services across all subspecialties with specific attention to the role of MIS during the recovery plan. Eighty-four of the statements were approved during the first round of Delphi voting (91.3\%) and another 8 during the following round after substantial modification, resulting in a $100 \%$ consensus.

Conclusion The recommendations formulated by the EAES board establish a framework for resumption of surgery following COVID-19 pandemic with particular focus on the role of MIS across surgical specialities. The statements have the potential for wide application in the clinical setting, education activities and research work across different healthcare systems.
\end{abstract}

Keywords COVID-19 $\cdot$ Delphi consensus $\cdot$ EAES guidance $\cdot$ Priority $\cdot$ Minimally invasive surgery

The rapid spread of the coronavirus disease 2019 (COVID19) presents an unprecedented crisis to the surgical community globally $[1,2]$. During the pandemic, elective surgical

The members of the EAES group of experts for Recovery amid COVID-19 pandemic have been included in the Acknowledgements Section.

Electronic supplementary material The online version of this article (https://doi.org/10.1007/s00464-020-08131-0) contains supplementary material, which is available to authorized users.

Alberto Arezzo

alberto.arezzo@unito.it

Extended author information available on the last page of the article practice was forced to rapidly decrease or even put on hold completely. As a consequence, there is a backlog of patients requiring surgical services but with limited human and institutional resources [3-8].

While health care systems are calling to resume elective surgical practice where pandemic is under control [1,2], uncertainty remains about the duration of the pandemic, the possibility of a second wave and the extent of its consequences on surgical services and patients $[9,10]$. This leaves the surgical community with unanswered clinical questions on patient and staff safety.

Various guidelines and recommendations have been published on issues pertaining to adapting surgical practice during the pandemic, often with conflicting recommendations, 
whereas many may not have been based on research evidence [11-14]. Preliminary guidelines recommended against performing laparoscopic surgery to avoid putative risks of SARS-CoV2 transmission via aerosolization due to pneumoperitoneum but were later revised recommending laparoscopic surgery under restrictions [15]. Several subsequent guidelines did not recommend against laparoscopic surgery; however, they advised strict precautions such as closed circuit smoke evacuation and the use of filtering system and personal protective equipment (PPE) [16-19]. The role of MIS has been argued to be more favourable in SARS-CoV-2 positive patients, as the potential benefits of MIS might exceed the risk of pneumoperitoneum on cardiovascular and respiratory systems [20].

Furthermore, there is substantial uncertainty with regard to specific questions related to laparoscopic procedures, and structured guidance pertinent to MIS procedures is lacking. There is an urgent need for practice-based recommendations in specific clinical situations with regard to safety precautions for patients and staff amidst the COVID-19 pandemic [21].

The purpose of this EAES initiative was to generate an updated and comprehensive set of management recommendations for MIS for each subspeciality of general surgery. This consensus was also designed to document the broader experts' opinion on how to optimize the use of human and institutional resources including the use of MIS techniques for patient benefit.

\section{Methods}

A steering group (SG) was formed comprising of six experts from the EAES Executive Board (AA, NF, YM, SM, DP and AP) who organized the project and guided the data synthesis. IRB approval and informed written consent were not required. The steps of the consensus process are outlined below.

\section{Identification of domains and formulation of questions}

The clinical questions were divided into eight domains: (i) general (ii) Hepatobiliary \& Pancreatic (HPB), (iii) Bariatrics (B), (iv) Abdominal Wall (AW), (v) Endocrine (E), (vi) Upper Gastrointestinal (UGI), (vii) Lower Gastrointestinal (LGI), (viii) technology and research. Domains (ii) to (vii) were divided into two subdomains: emergency and elective.

\section{Identification of experts to address questions}

Fifty-Five out of EAES board members accepted the task to contribute to this project and were divided into eight subgroups organized by the domain topics and led by a designated chair (Table 1). In addition, key stakeholders were invited to provide expert input into the multidisciplinary aspects of this project including anaesthesiology, radiology and oncology and were allocated to a relevant group, based on their expertise.

\section{Search methods and inclusion criteria}

Based on the research questions, a literature search was designed and performed by two independent EAES experts (AA, NF). The PubMed and Embase databases were queried for articles published before May 10th, 2020. Inclusion criteria were systematic and narrative reviews, commentaries, randomized clinical trials, cohort studies and case series on the subject of surgery during the COVID-19 and other pandemic published in the English language. Search syntaxes used was (COVID OR 'SARS CoV 2' OR coronavirus AND surgery).

Table 1 Expert group members and topic allocations

\begin{tabular}{|c|c|c|}
\hline Topic & Leader & Experts \\
\hline General introduction & Catalin Copaescu & $\begin{array}{l}\text { Emina Letić, Silviu Daniel Preda, Alice Tsai, Ewelina Malanowska, Dusan Lesko, Wlodzimierz } \\
\text { Majewski, Ludovica Baldari }\end{array}$ \\
\hline Hepatobiliary & Nicolò de Manzini & Luca Morelli, Andreas Shamiyeh, Gil Faria \\
\hline Bariatrics & Nicola Di Lorenzo & Francesco Maria Carrano, Piotr Mysliwiec, Gunnar Ahlberg \\
\hline Abdominal Wall & Stavros Antoniou & Elisa Cassinotti, Samir Delibegović, Lubomír Martinek \\
\hline Endocrine & Nicole Bouvy & Eugenia Yiannakopoulou, Marguerite Gorter-Stam, Hendrik Jaap Bonjer \\
\hline Upper GI & $\begin{array}{l}\text { Beat P. Müller- } \\
\text { Stich }\end{array}$ & $\begin{array}{l}\text { George Hanna, Hans Fuchs, Miloš Bjelovic, Sheraz Markar, Philip Wai Yan, Chiu, Bang Wool } \\
\text { Eom, Young-Woo Kim, Carmen Balagué Ponz, Marlies Schijven }\end{array}$ \\
\hline Lower GI & Michel Adamina & $\begin{array}{l}\text { Luigi Boni, Thomas Carus, George Theodoropoulos, Antonello Forgione, Marco Milone, Wanda } \\
\text { Luisa Rita Petz, Predag Andrejevic, Dejan Ignjatovic, Thanjakumar Arulampalam, Kenneth } \\
\text { Campbell, Manish Chand, Mark Coleman, Christos Kontovounisios }\end{array}$ \\
\hline Technology \& Research & Felix Nickel & $\begin{array}{l}\text { Chen Sagiv, Fanny Ficuciello, Stefania Marconi, Pietro Mascagni, Kiyokazu Nakajima, Francisco } \\
\text { Miguel Sánchez Margallo, Tim Horeman, George Mylonas, Pietro Valdastri }\end{array}$ \\
\hline
\end{tabular}




\section{Formulation of questions and statements}

Questions were drafted and submitted to the steering group, which approved them prior to sending them to the subgroups to formulate statements in response to these questions. Statements were generated by each subgroup in each topic question of their domain. Each group was advised to include any available evidence to support their statements and when evidence was not available, experts' opinion was considered. Each group conducted a literature research and drafted statements and recommendations in response to their research questions. The literature review was reported in accordance with PRISMA statement standards for systematic reviews and meta-analyses [22]. Statements were then submitted to the steering group (SG) who did not participate in the formulation of these statements or in the voting process. After structural editing by the SG group, all questions were unanimously approved by all experts (Table 1).

\section{Voting and data analysis}

The statements received from each subgroup of experts was compiled by the SG and a modified Delphi methodology process was followed to reach agreement among all the experts on all statements and recommendations [23].

EAES board members voted to declare agreement or disagreement with the statements using closed-ended questions (agree or disagree), whereas there was an option to submit free text comments. The Delphi process was implemented through the SurveyMonkey electronic platform (https://nl.surveymonk ey.com). To reduce the possibility of bias among participants, the authors of the statements and the resultant votes/comments remained anonymous. Each statement was subjected to live voting by all experts including key stakeholders and excluding the steering committee.

Consensus was achieved when a statement reached at least $70 \%$ expert agreement. Statements with less than $70 \%$ agreement in the first round were returned to the expert subgroup who modified them according to the comments. The subgroups had the option to revise statements based on feedback for further voting.

The results of the consensus are summarized using descriptive statistics. The manuscript was then drafted with the recommendations following completion of all voting and statement formulation and sent to all members for revision, input and approval prior to submission for publication.

\section{Results}

Ninety-two statements were generated by the subgroups across the eight topics. The full text literature analyses and references used to generate statements and recommendations for all topics are included as Appendix 1.

Eighty-four out of 92 statements (91\%) were approved in the first round and further 8 modified statements were approved in the second round.

Overall, the Delphi process approved 92 statements $(100 \%)$ for the consensus guidelines. The questions, final recommendations and respective approval rates in each step of the Delphi processes are summarized in Table 2.

Initial disagreement was regarded priorities depending on local resources, the use of energy dissection devices and the risk of aerosolization, the indication for stenting for obstructed colorectal cancer, the indication of laparoscopic lavage for diverticular disease, the indication of neoadjuvant treatment for early rectal cancer in order to postpone surgery, the management of undiagnosed pancreatic lesions, the indication for intragastric balloons to postpone bariatric surgery and the indication for endoscopic therapies for achalasia and reflux disease to postpone surgery.

\section{Discussion}

The study achieved its objective of formulating EAES evidence-based recommendations to provide guidance on the resumption of MIS across various general surgery specialities, taking into account the serious burden on our healthcare systems caused by the COVID-19 pandemic. These statements provided descriptive safety guidance measures that should be undertaken in the recovery plan for elective and emergency surgery across all subspecialties with specific measures for MIS.

In a recent survey, over 28 million patients are awaiting treatment worldwide, a number which continues to grow in the setting of new restrictions on delivery of care and a pandemic that is still evolving [24]. As this progression is expected to continue, and given the uncertainty about the ongoing pandemic, adaptive changes are required in procedure-based specialties to include safety, logistic, service relocation, economic and ethical considerations [25-30].

Through this project, consensus was achieved on all the proposed statements by the expert across the different domains, providing specific guidance on how to safely resume MIS and implement adaptive changes in procedure specific manner.

This project adhered to Delphi principles dictating anonymous voting. The selection of the steering group 


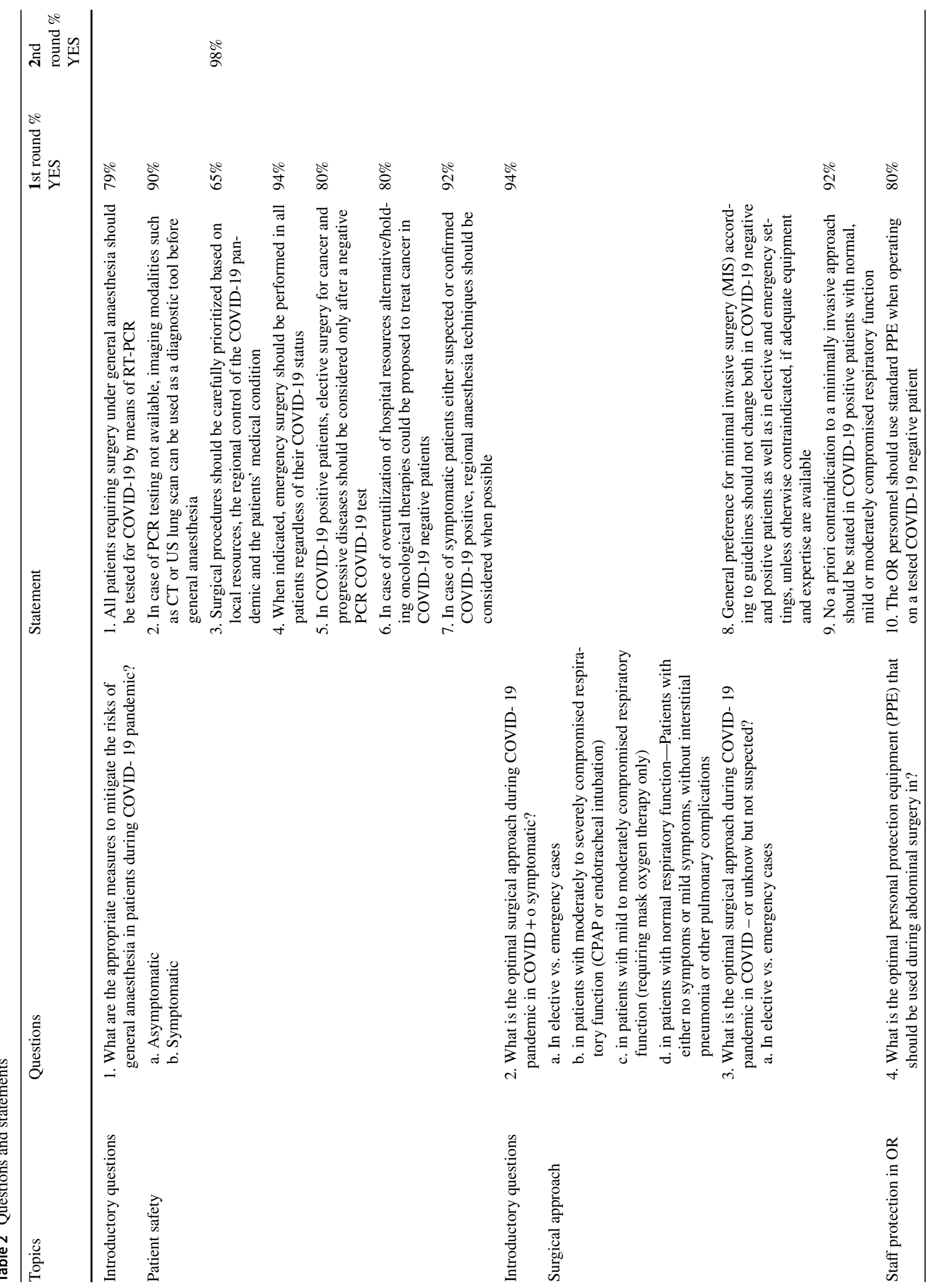




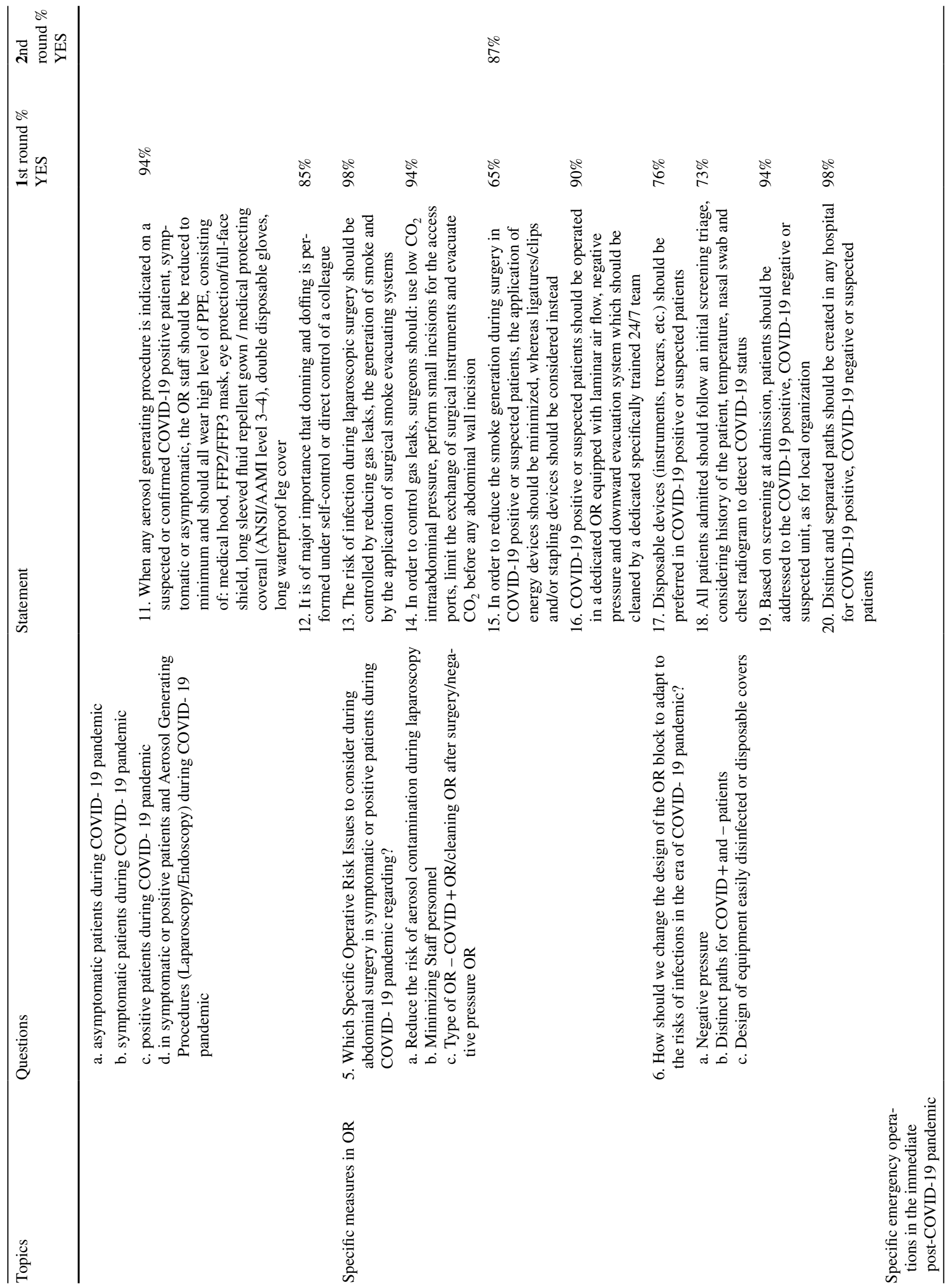




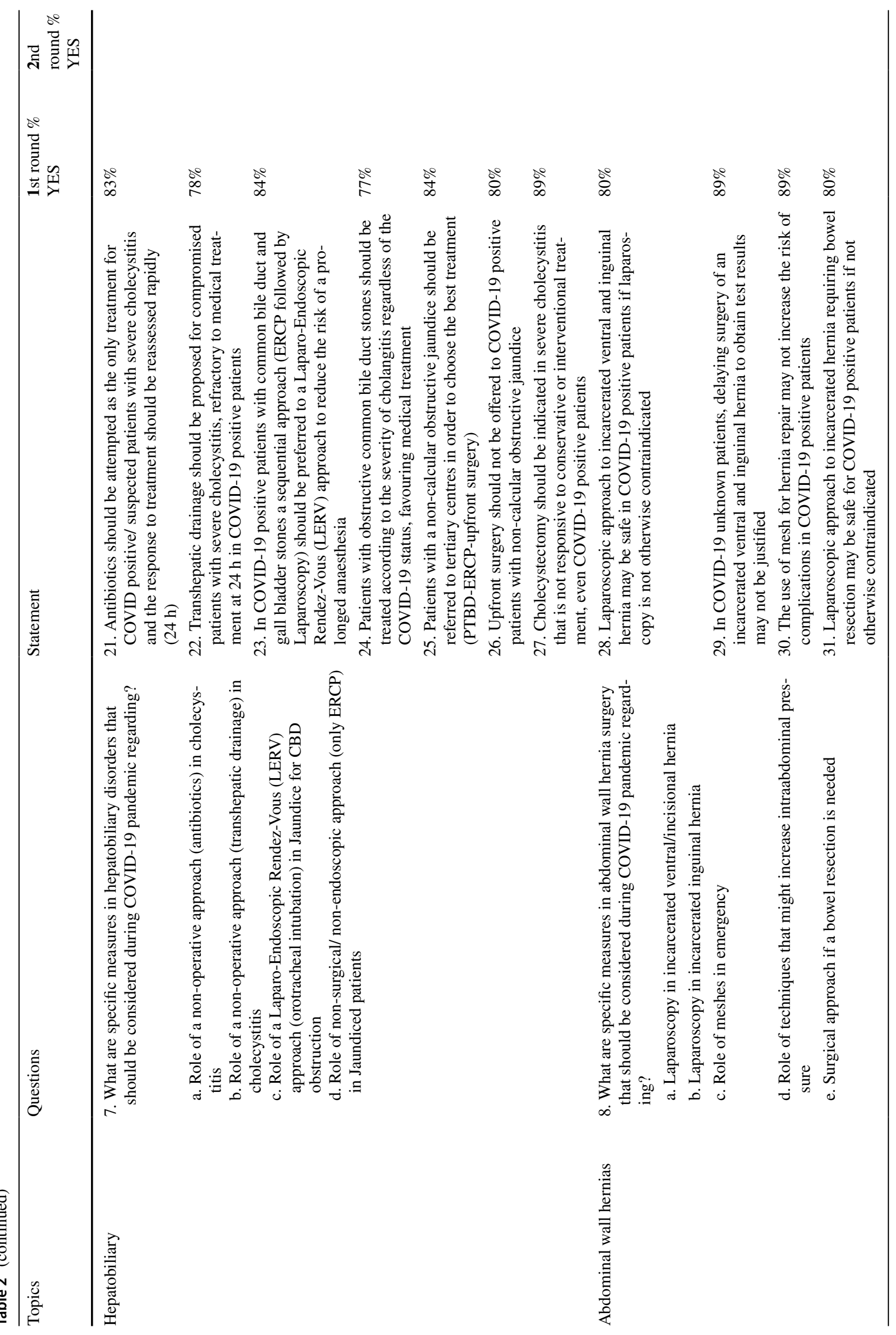




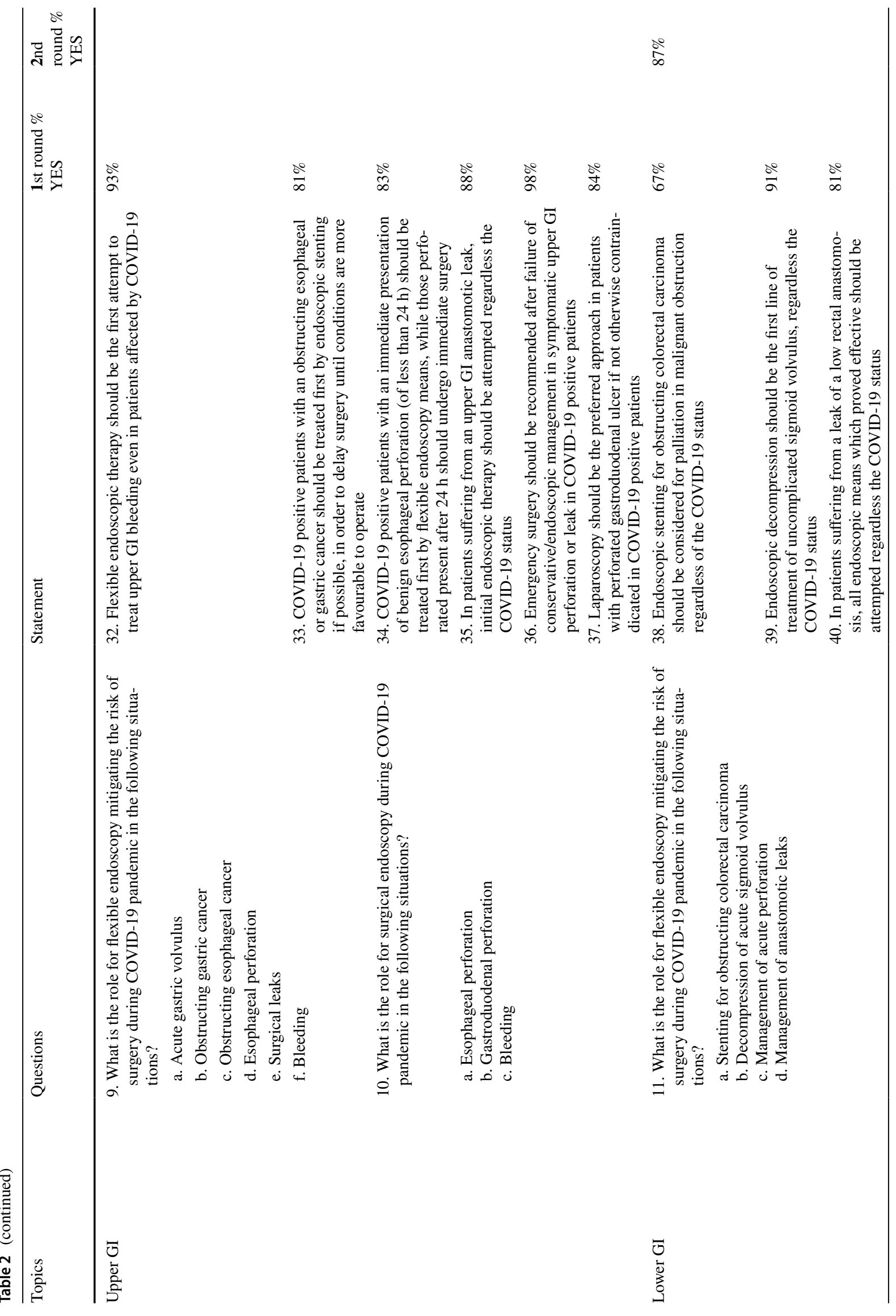




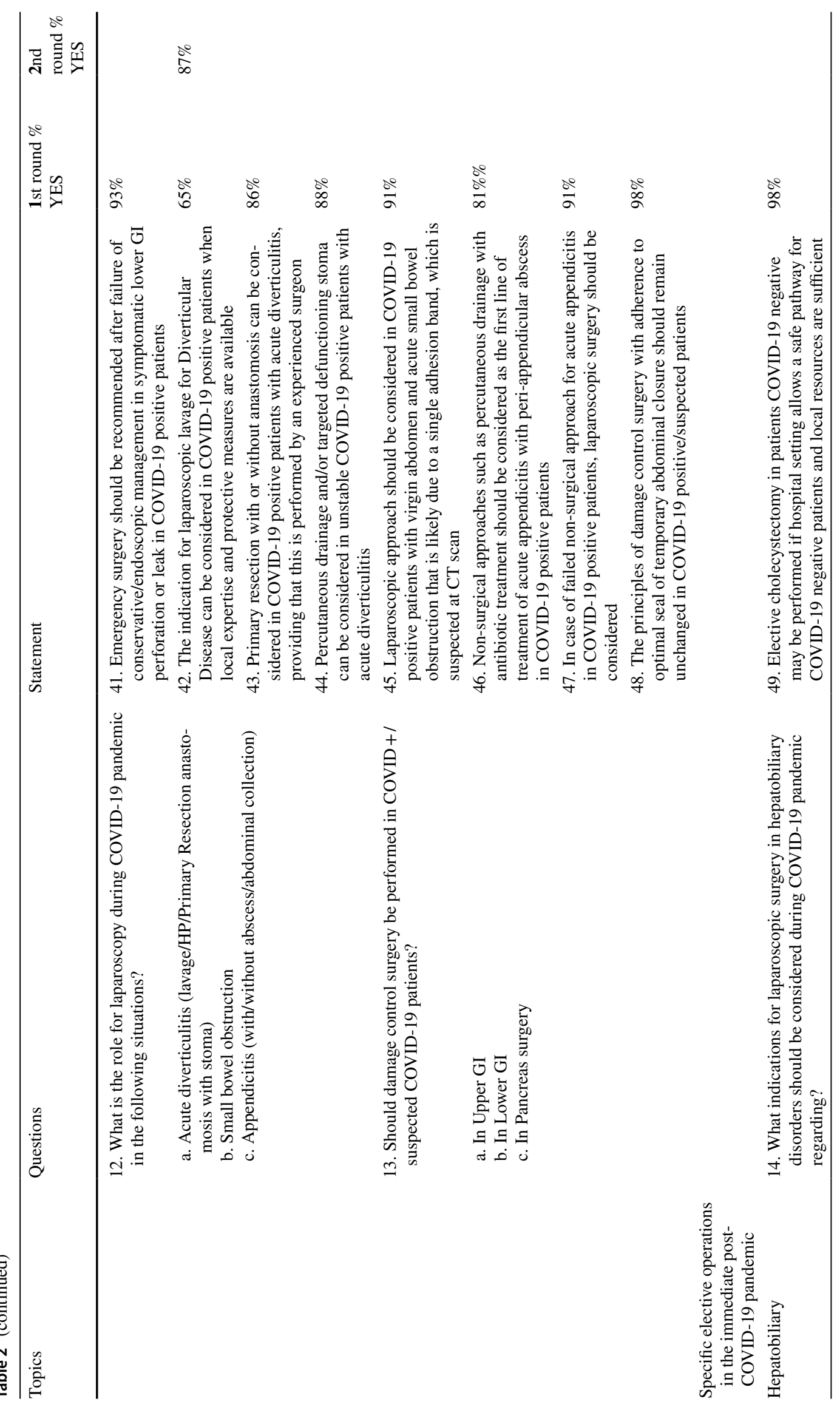




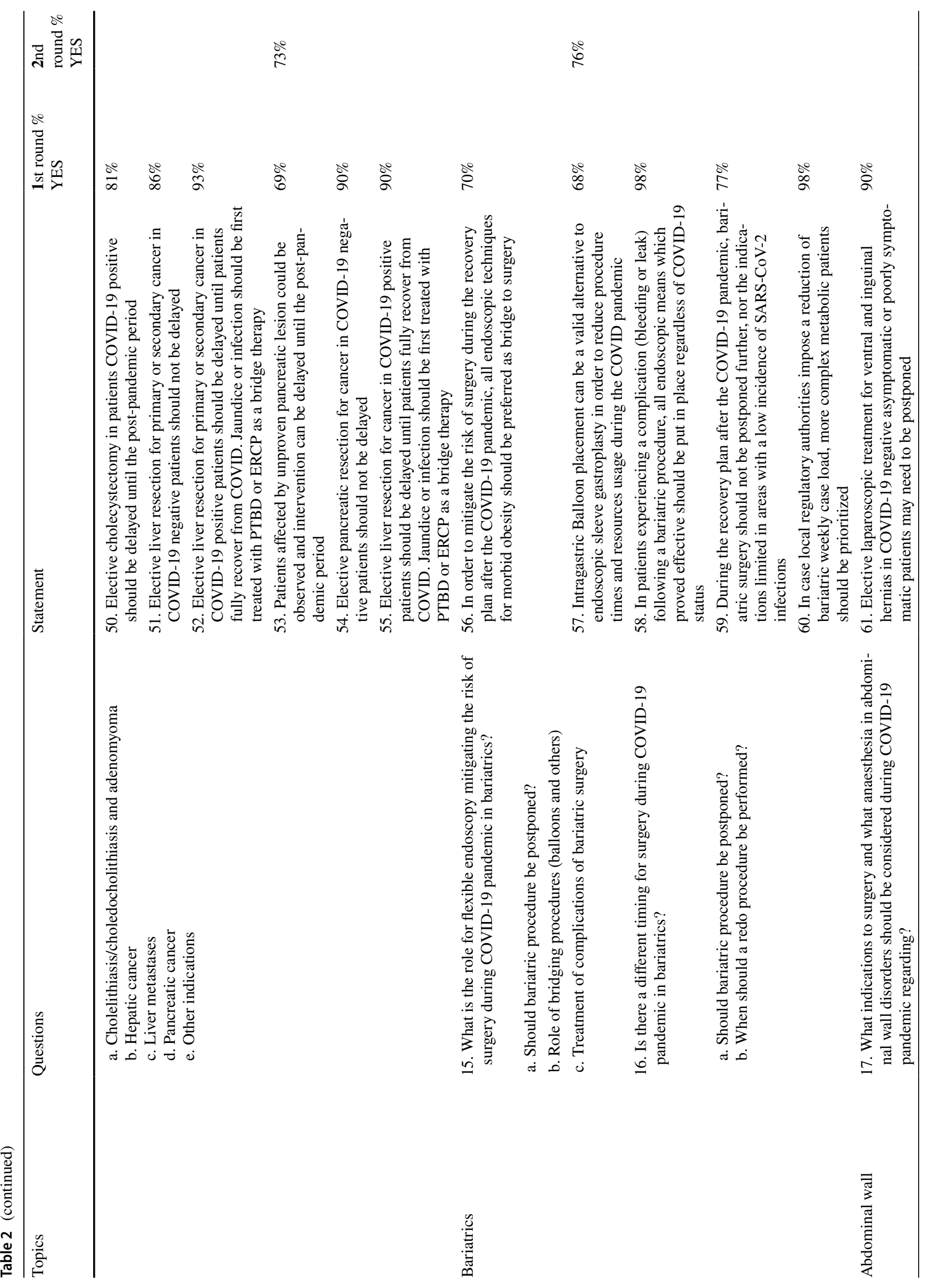




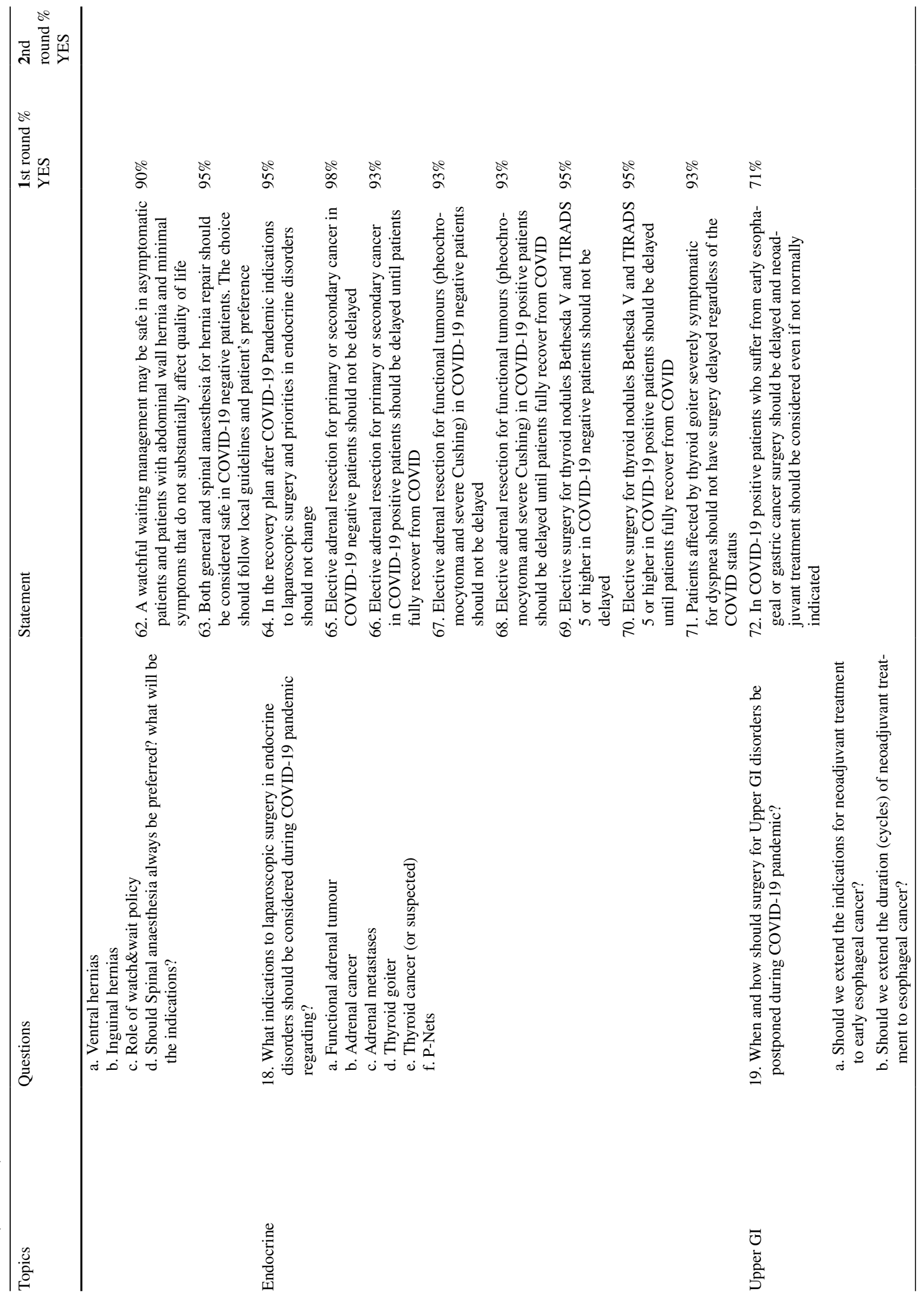




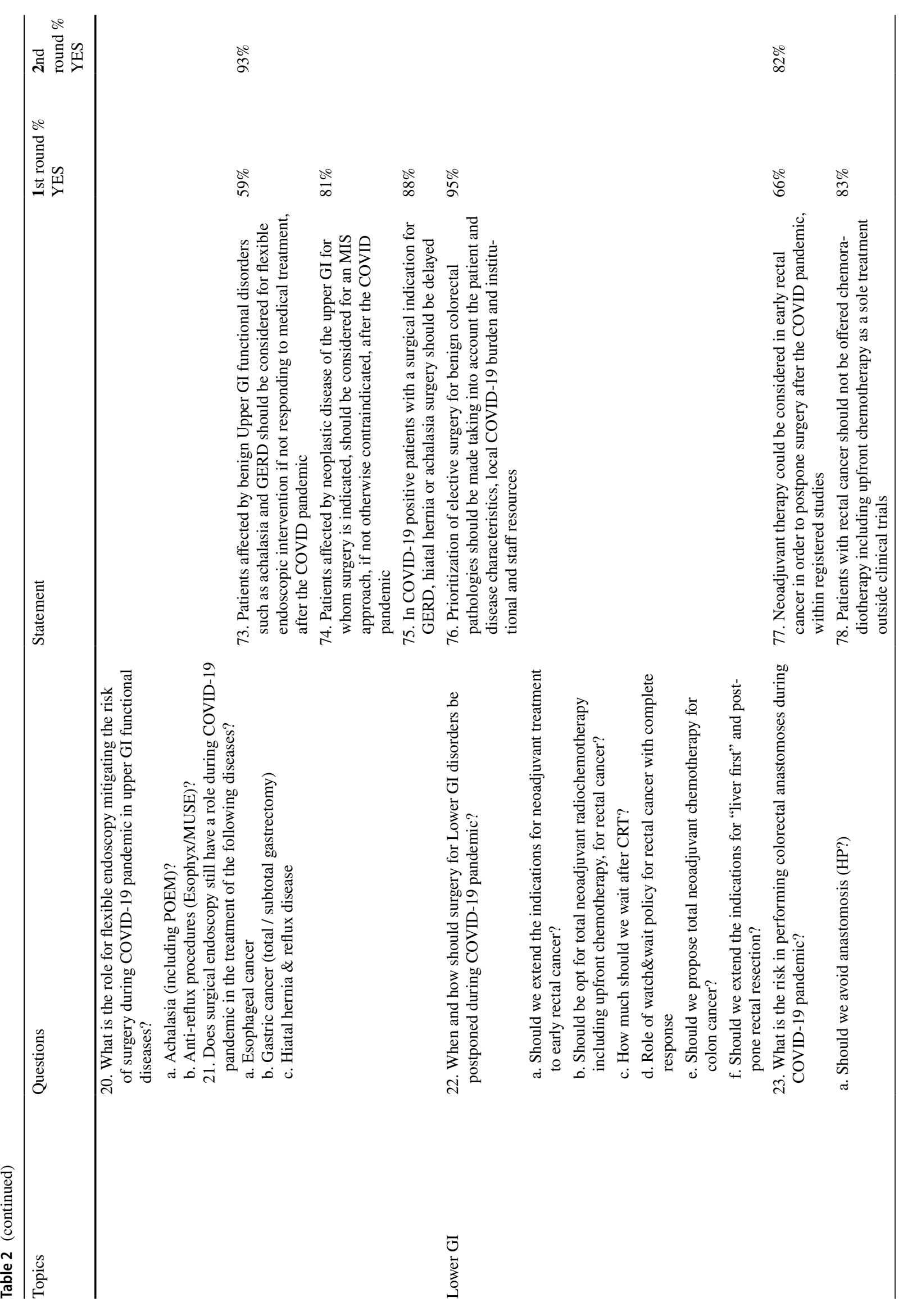




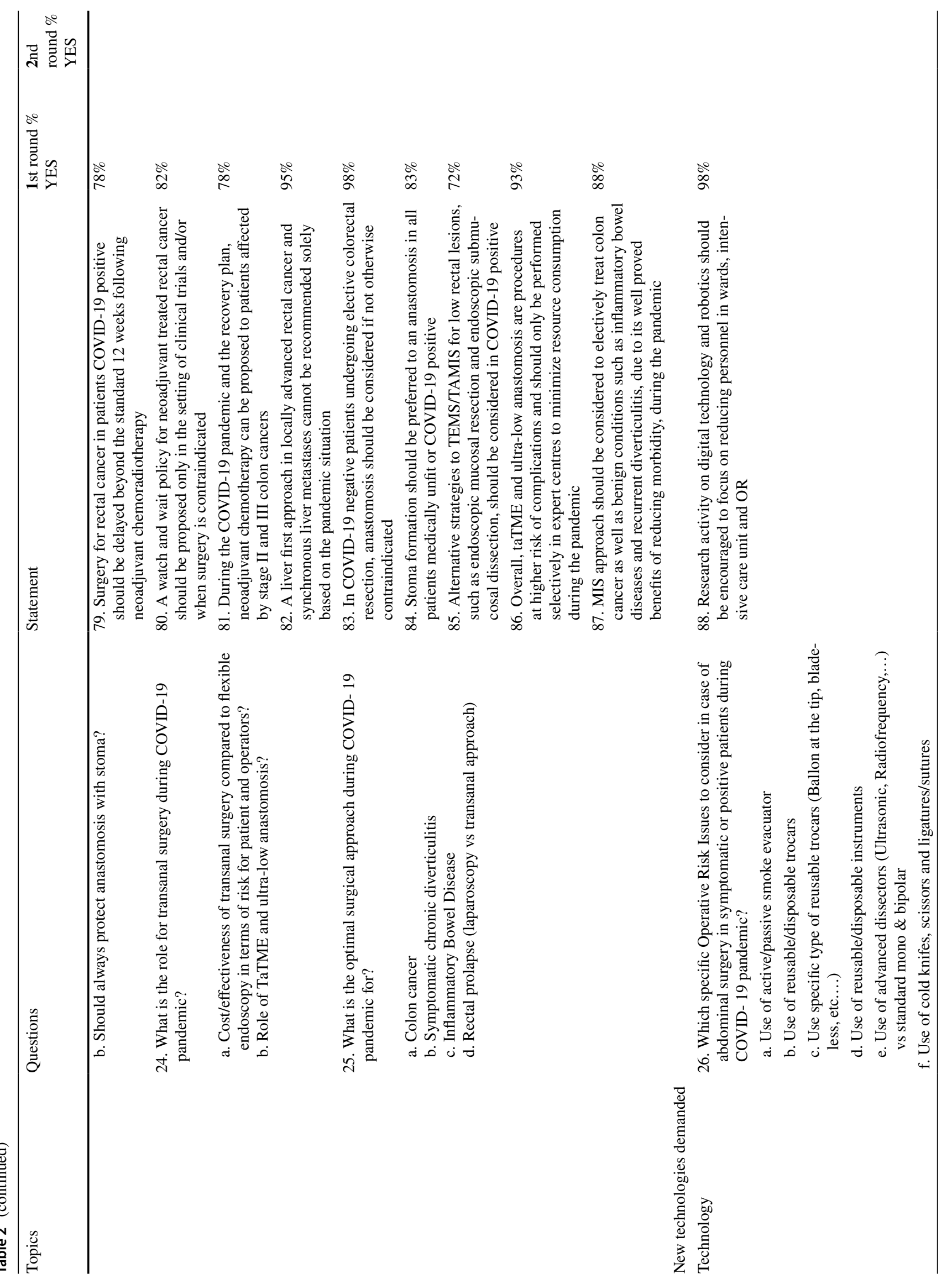




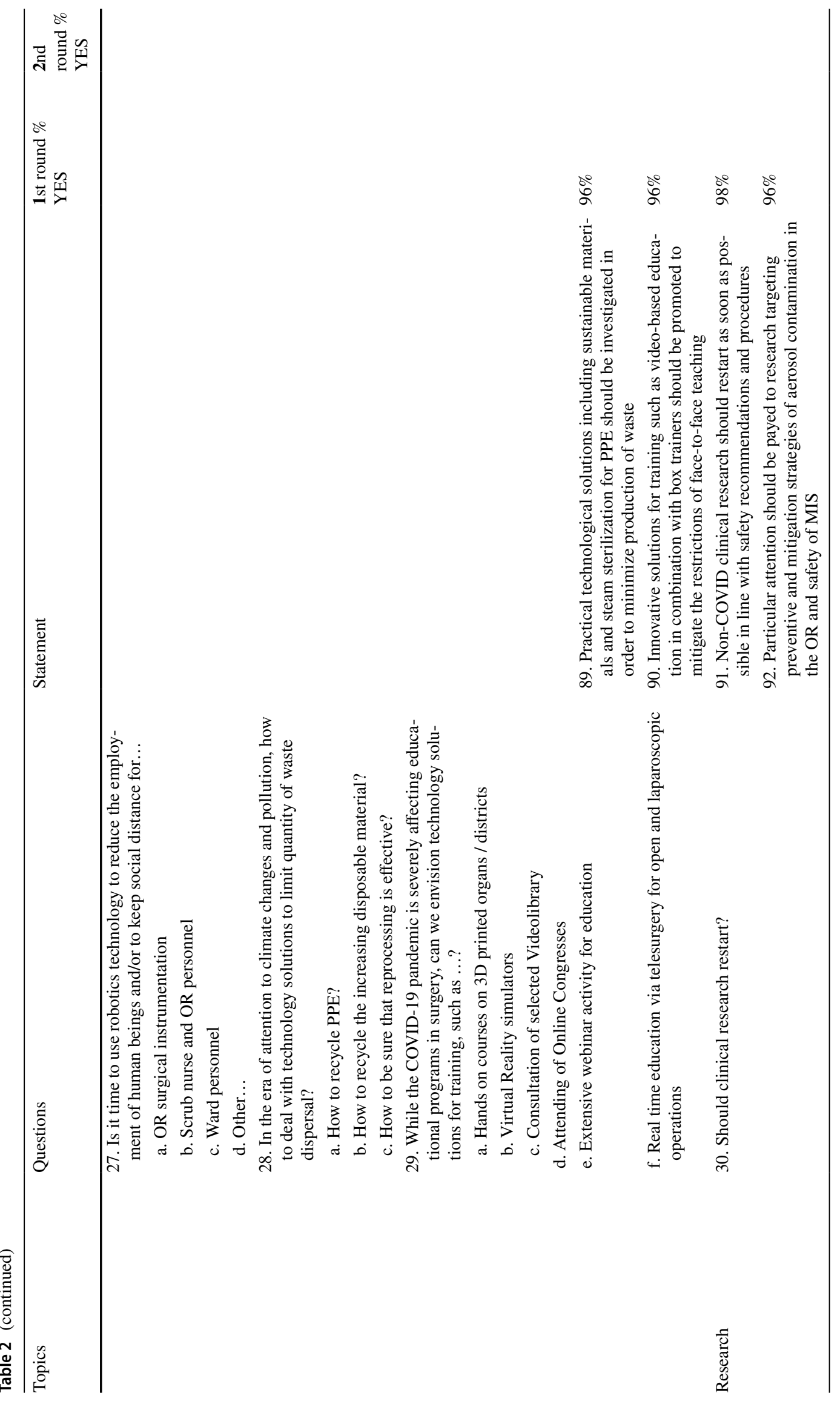


and the domains chairs was based on their peers' recommendations and their leadership positions across different specialties and of their expertise on the research methodology of consensus development. The steering group did not contribute to the voting process.

The Delphi design allowed us to elicit the opinion of the EAES board members along with additional key stake holders to complement the multidisciplinary and heterogeneous nature of the international panel of experts. Although evidence synthesis was part of this project to generate evidencebased recommendations, there was no found evidence that can inform the statements, hence, relied on expert opinion.

A limited number of areas of continuing controversy were identified at the first voting round with lack of consensus among members. Initial disagreement was encountered on how to prioritize surgery, but ultimately total agreement was achieved by recommending that decisions should be based on local resources, the regional control of the COVID-19 pandemic and the patients' medical condition. This was fundamental in deciding priorities also hereinafter for the rest of the consensus, but it is probably an area that requires fundamental research in the immediate future.

While there was also general agreement on the need to screen all patients undergoing surgery under general anaesthesia, by Reverse Transcription Polymerase Chain Reaction (RT-PCR) or even by computer tomography (CT) or ultrasound (US) lung scan in symptomatic patients, the option of regional anaesthesia should always be considered in suspected or positive patients for whom surgery cannot be postponed. Elective oncologic surgery should be only offered to SARS-CoV-2 negative patients or to previously positive patients after conversion to a negative RT-PCR COVID-19 test.

One of the main objectives of this project was to clarify the role of MIS, considering the conflicting information from different guidelines. This was based on the theoretical risk of possible contamination due to aerosolization and the gas leaks demonstrated during laparoscopy [31-33].

Until high-level evidence will be available to provide an answer about the direct link between SARS-CoV-2 contamination by pneumoperitoneum and its contagion to the operating team, the application of MIS across all surgical specialities has been supported by the experts in this project, provided local expertise is available and safety procedures are adhered to. Additionally, there was wide agreement that the general preference for MIS according to guidelines should not depend on the SARS-CoV-2 status or the indication of surgery in terms of elective and emergency settings.

These precautions are in line with the recent EAES/ SAGES recommendations to reduce gas leaks [34], the generation of smoke and by the use of surgical smoke evacuating systems [16]. These guidelines are also supported by the argument that containing potentially contaminated gas within a defined space, as it happens during laparoscopy, should provide a better control of risk, when compared to open surgery [11]. Regardless to the mode of surgery, limiting the use of energy devices in SARS-CoV-2 positive patients was also recommended and favouring ligatures/clips and/or stapling devices when possible. This is also in line with other recommendations (EAES/SAGES) and with evidence suggesting gas escape through trocars [30-32]. General recommendations for personnel safety in the OR including characteristics of the environment and PPE were also confirmed [16].

In the emergency setting, a number of recommendations were proposed by the experts, supporting conservative treatment for abscesses and collections in SARS-CoV-2 positive patients, rather than offering immediate surgery, if the general condition of patients allows this. On the contrary, for acute cholecystitis in SARS-CoV-2 positive patients, cholecystectomy was recommended when not responsive within $24 \mathrm{~h}$ rather than interventional treatment such as percutaneous transhepatic drainage, with the exception of ASA 3 and 4 patients.

In the field of abdominal wall surgery, laparoscopy was recommended for incarcerated ventral and inguinal hernia if not otherwise contraindicated and should not be postponed if clinically indicated. Emergency endoscopy (diagnostic and therapeutic) was supported in SARS-CoV-2 positive patients, as the first line to assess and possibly to treat bleeding, neoplastic obstruction, perforation and anastomotic leak. Similarly, laparoscopic surgery should be considered after failure of conservative/endoscopic management in symptomatic patients, as well as the in acute diverticulitis management according to the accepted algorithms.

Elective surgery for both malignant and benign disease should be postponed in SARS-CoV-2 positive patients until they return negative. This is also the case for hepatobiliary and pancreatic non-neoplastic diseases, as well as other oncologic patients, in whom interim procedures should be offered instead. For instance, drainage of the biliary tract by Percutaneous Transhepatic Biliary Drainage (PTBD) or Endoscopic Retrograde Cholangio Pancreatography (ERCP) should be considered as a bridging therapy.

In the field of bariatric surgery, the expert group in this project supported postposing elective surgery until the recovery plan, and flexible endoscopic procedures such as intragastric balloons was recommended as a bridge to surgery during the COVID-19 pandemic. Elective laparoscopic treatment for ventral and inguinal hernia in SARS-CoV-2 negative patients may need to be postponed depending on the local situation. Endocrine surgery should only be cancelled in SARS-CoV-2 positive patients until they convert to negative. Otherwise, patients should be prioritized depending on symptoms and oncological risk. 
For upper and lower gastrointestinal cancer, neoadjuvant therapy could be considered for early cancers in order to postpone surgery after the COVID-19 pandemic, but only within registered studies, although difficult to arrange in short time. Functional disorders such as achalasia and reflux disease should be treated as usual and surgery can be considered if not responding to conservative treatment. The experts recommend that surgery should be delayed only in SARSCoV-2 positive patients. Similarly, elective surgical treatment of benign colorectal pathologies should be prioritized based on patient and disease characteristics, local COVID-19 burden and institutional and staff resources. If not otherwise contraindicated, colorectal resections should be completed with anastomosis, while stoma formation should be applied as usual only for high-risk patients. Particular attention should be paid when Transanal Endoscopic Microsurgery (TEMS) or TransAnal Minimally Invasive Surgery (TAMIS) procedures are indicated, including TransAnal Total Mesorectal Excision (TaTME), due to the particularly high risk of operator contamination. In fact, alternative strategies for low rectal lesions, such as endoscopic mucosal resection and endoscopic submucosal dissection, should be considered in SARS-CoV-2 positive. On the other hand, due to its well proved benefits, a minimally invasive approach should be considered to treat colorectal cancer as well as benign conditions such as inflammatory bowel diseases and recurrent diverticulitis.

Finally, we focused on technology, education and research in the time of pandemic. The team of experts outlined how research activity on digital technology and robotics should be encouraged to focus on reducing the numbers of working personnel in wards, intensive care unit and the operating rooms. In fact, this pandemic highlighted the importance of technology advancement in remote teaching and mentorship. Innovative solutions for training such as video-based education in combination with box trainers should be promoted to mitigate the restrictions of face-to-face teaching. The experts outlined certain areas of further research targeting robotics, Artificial Intelligence, advanced imaging and energy devices that could have a positive impact in times of pandemic and restrictions due to social distancing. At the same time practical technological solutions including sustainable materials and steam sterizilation for PPE should be investigated in order to minimize production of waste.

Overall, this project highlighted interesting trends and controversies related to surgeons' willingness to overcome this difficult time, but it holds a number of limitations. There is a lack of empirical data to support many of the underlying statements, hence weaknesses inherent to these guidelines include the reliance on expert opinion and discussion to formulate recommendations. Despite the limited evidence, this project highlighted a number of clinically relevant questions that provide an agenda to stimulate future research in this field. The selection of experts is another critical aspect within consensus statements development. The expert group involved in this research were all the EAES board members representing the research, technology and educational committees as well as the members of the executive committee of the society. The response rate among the participants was high across the entire process reflecting the hard work and commitment of the board members to undertake this project and complete the project in a timely manner given the urgency and the need for the guidelines.

\section{Conclusion}

The recommendations formulated by the EAES board create a framework for resumption of surgery following COVID-19 pandemic with particular focus on the role of MIS across all specialities. The statements have the potential for wide application in clinical setting, education and research across different healthcare systems.

Acknowledgements We would like to thank Muriel Van Eck and the entire EAES Office for supporting us in this survey activity.

Members of the EAES group of experts for Recovery amid COVID19 pandemic: Emina Letić, Silviu Daniel Preda, Alice Tsai, Ewelina Malanowska, Dusan Lesko, Wlodzimierz Majewski, Ludovica Baldari, Luca Morelli, Andreas Shamiyeh, Gil Faria, Francesco Maria Carrano, Piotr Mysliwiec, Gunnar Ahlberg, Elisa Cassinotti, Samir Delibegović, Lubomír Martinek, Eugenia Yiannakopoulou, Marguerite Gorter-Stam, Hendrik Jaap Bonjer, George Hanna, Hans Fuchs, Miloš Bjelovic, Sheraz Markar, Philip Wai Yan, Chiu, Bang Wool Eom, Young-Woo Kim, Carmen Balagué Ponz, Marlies Schijven, Luigi Boni, Thomas Carus, George Theodoropoulos, Antonello Forgione, Marco Milone, Wanda Luisa Rita Petz, Predag Andrejevic, Dejan Ignjatovic, Thanjakumar Arulampalam, Kenneth Campbell, Manish Chand, Mark Coleman, Christos Kontovounisios, Chen Sagiv, Fanny Ficuciello, Stefania Marconi, Pietro Mascagni, Kiyokazu Nakajima, Francisco Miguel Sánchez Margallo, Tim Horeman, George Mylonas, Pietro Valdastri.

Funding Open access funding provided by Università degli Studi di Torino within the CRUI-CARE Agreement.

\section{Compliance with ethical standards}

Disclosures Alberto Arezzo has financial disclosures unrelated to this manuscript being consultant for Karl Storz, BBraun and Medrobotics. Nader Francis has financial disclosures unrelated to this manuscript being consultant for Pharmacozmos, Fisher \& Paykel Healthcare, Medtronic and Olympus. Yoav Mintz, Michel Adamina, Stavros A Antoniou, Nicole Bouvy, Catalin Copaescu, Nicolò de Manzini, Nicola Di Lorenzo, Salvador Morales-Conde, Beat P Müller-Stich, Felix Nickel, Dorin Popa, Diana Tait, Cenydd Thomas, Susan Nimmo Dimitrios Paraskevis Andrea Pietrabissa have no conflict of interest or financial ties to disclose.

Open Access This article is licensed under a Creative Commons Attribution 4.0 International License, which permits use, sharing, adaptation, distribution and reproduction in any medium or format, as long as you give appropriate credit to the original author(s) and the source, 
provide a link to the Creative Commons licence, and indicate if changes were made. The images or other third party material in this article are included in the article's Creative Commons licence, unless indicated otherwise in a credit line to the material. If material is not included in the article's Creative Commons licence and your intended use is not permitted by statutory regulation or exceeds the permitted use, you will need to obtain permission directly from the copyright holder. To view a copy of this licence, visit http://creativecommons.org/licenses/by/4.0/.

\section{References}

1. AHA Letter to Surgeon General Re: Elective Surgeries and COVID-19 | AHA. Am. Hosp. Assoc. https://www.aha.org/lette rcomment/2020-03-15-aha-letter-surgeon-general-re-electivesurgeries-and-covid-19. Accessed 26 May 2020

2. Evans M, Mathews AW. Hospitals Push Off Surgeries to Make Room for Coronavirus Patients - WSJ. https://www.wsj.com/artic les/hospitals-push-off-surgeries-to-make-room-for-coronaviru s-patients-11584298575. Accessed 26 May 2020

3. Joint Statement: Roadmap for Resuming Elective Surgery after COVID-19 Pandemic. Am. Coll. Surg. https://www.facs.org/covid -19/clinical-guidance/roadmap-elective-surgery. Accessed 18 May 2020

4. CMS Issues Recommendations to Re-Open Health Care Systems in Areas with Low Incidence of COVID-19 I CMS. Cent. Medicare Medicaid Serv. https://www.cms.gov/newsroom/press-relea ses/cms-issues-recommendations-re-open-health-care-systemsareas-low-incidence-covid-19. Accessed 26 May 2020

5. Yoon DH, Koller S, Duldulao PMN, Ault GT, Lee SW, Cologne KG (2020) J COVID-19 Impact on Colorectal Daily Practice-How Long Will It Take to Catch Up? Gastrointest Surg. https://doi. org/10.1007/s11605-020-04722-3

6. Ding A, Onida S, Davies AH (2020) The painful cost of cancelling surgery due to COVID-19- can we do anything about it? Br J Surg. 107(9):e336. https://doi.org/10.1002/bjs.11786

7. Soreide K, Hallet J, Matthews JB et al (2020) Immediate and longterm impact of the COVID-19 pandemic on delivery of surgical services. Br J Surg. https://doi.org/10.1002/bjs.11670

8. COVIDSurg Collaborative (2020a) Global guidance for surgical care during the COVID-19 pandemic. Br J Surg. https://doi. org/10.1002/bjs.11646

9. Abu Hilal M, Besselink MG, Lemmers DHL, Taylor MA, Triboldi A (2020) Early look at the future of healthcare during the COVID19 pandemic. Br J Surg. https://doi.org/10.1002/bjs.11666

10. COVIDSurg Collaborative (2020b) Elective surgery cancellations due to the COVID-19 pandemic: global predictive modelling to inform surgical recovery plans. Br J Surg. https://doi.org/10.1002/ bjs. 11746

11. Mintz Y, Arezzo A, Boni L, Baldari L, Cassinotti E, Brodie R, Uranues S, Zheng M, Fingerhut A (2020) The risk of COVID-19 transmission by laparoscopic smoke may be lower than for laparotomy: a narrative review. Surg Endosc 34(8):3298-3305. https ://doi.org/10.1007/s00464-020-07652-y (Epub 2020 May 26)

12. Chadi SA, Guidolin K, Caycedo-Marulanda A, Sharkawy A, Spinelli A, Quereshy FA, Okrainec A (2020) Current evidence for minimally invasive surgery during the COVID-19 pandemic and risk mitigation strategies: a narrative review. Ann Surg 272(2):e118-e124

13. Ramos RF, Lima DL, Benevenuto DS (2020) Recommendations of the Brazilian College of Surgeons for laparoscopic surgery during the COVID-19 pandemic. Rev Col Bras Cir 3(47):e20202570

14. Campanile FC, Podda M, Arezzo A, Botteri E, Sartori A, Guerrieri M, Cassinotti E, Muttillo I, Pisano M, Brachet Contul R,
D’Ambrosio G, Cuccurullo D, Bergamini C, Allaix ME, Caracino V, Petz WL, Milone M, Silecchia G, Anania G, Agrusa A, Di Saverio S, Casarano S, Cicala C, Narilli P, Federici S, Carlini M, Paganini A, Bianchi PP, Salaj A, Mazzari A, Meniconi RL, Puzziello A, Terrosu G, De Simone B, Coccolini F, Catena F (2020) Agresta F Acute cholecystitis during COVID-19 pandemic: a multisocietary position statement. World J Emerg Surg 15(1):38

15. Royal College of Surgeons of England. Updated intercollegiate general surgical guidance on COVID-19. https://www.rcseng.ac. uk/coronavirus/joint-guidance-for-surgeons-v2 (cited April 2020).

16. Francis N, Dort J, Cho E, Feldman L, Keller D, Lim R, Mikami D, Phillips E, Spaniolas K, Tsuda S, Wasco K, Arulampalam T, Sheraz M, Morales S, Pietrabissa A, Asbun H, Pryor A (2020) SAGES and EAES recommendations for minimally invasive surgery during COVID-19 pandemic. Surg Endosc 34(6):2327-2331. https://doi.org/10.1007/s00464-020-07565-w (Epub 2020 Apr 22)

17. Moletta L, Pierobon ES, Capovilla G, Costantini M, Salvador R, Merigliano S, Valmasoni M (2020) International guidelines and recommendations for surgery during Covid-19 pandemic: a systematic review. Int J Surg 79:180-188

18. Shabbir A, Menon RK, Somani J, So JBY, Ozman M, Chiu PWY, Lomanto D (2020) ELSA recommendations for minimally invasive surgery during a community spread pandemic: a centered approach in Asia from widespread to recovery phases. Surg Endosc 34(8):3292-3297

19. American College of Surgeons (2020) COVID-19 Guidelines for Triage of Emergency General Surgery Patients. https://www.facs. org/covid-19/clinical-guidance/elective-case/emergency-surgery. Accessed 26 March 2020

20. Mintz Y, Arezzo A, Boni L, Baldari L, Cassinotti E, Brodie R, Uranues S, Zheng MH, Fingerhut A (2020, in press) Minimally Invasive Surgery is the Key to Patient and Operating room team Safety During the COVID19 Pandemic as well as in the "new normal" or chronic Pandemic State to come. Br J Surg

21. COVIDSurg Collaborative (2020c) Global guidance for surgical care during the COVID-19 pandemic. Br J Surg. https://doi. org/10.1002/bjs. 11646

22. Moher D, Liberati A, Tetzlaff J, Altman DG, The PRISMA Group (2009) Preferred reporting items for systematic reviews and metaanalyses: the PRISMA statement. PLoS Med 6(7):e1000097. https ://doi.org/10.1371/journal.pmed.1000097

23. Gorter RR, Eker HH, Gorter-Stam MA, Abis GS, Acharya A, Ankersmit $M$ et al (2016) Diagnosis and management of acute appendicitis. EAES consensus development conference. Surg Endosc 30(11):4668-4690

24. Nepogodiev D, Bhangu A. Elective surgery cancellations due to the COVID-19 pandemic: global predictive modelling to inform surgical recovery plans. BJS Br J Surg; n/a. DOI:https://doi. org/10.1002/bjs.11746.

25. Søreide K, Hallet J, Matthews JB et al (2020) Immediate and longterm impact of the COVID-19 pandemic on delivery of surgical services. Br J Surg. https://doi.org/10.1002/bjs.11670

26. Emanuel EJ, Persad G, Upshur R et al (2020) Fair allocation of scarce medical resources in the time of Covid-19. N Engl J Med 382:2049-2055

27. Khullar D, Bond AM, Schpero WL (2020) COVID-19 and the financial health of US hospitals. JAMA. https://doi.org/10.1001/ jama.2020.6269

28. Spinelli A, Pellino G (2020) COVID-19 pandemic: perspectives on an unfolding crisis. Br J Surg. https://doi.org/10.1002/ bjs. 11627

29. Brindle ME, Doherty G, Lillemoe K, Gawande A (2020) Approaching surgical triage during the COVID-19 pandemic. Ann Surg. https://doi.org/10.1097/SLA.0000000000003992 
30. Milone M, Carrano FM, Letić E et al (2020) Surgical challenges and research priorities in the era of the COVID-19 pandemic: EAES membership survey. Surg Endosc. https://doi.org/10.1007/ s00464-020-07835-7

31. Dalli J, Khan MF, Nolan K, Cahill RA (2020) Laparoscopic pneumoperitoneum escape and contamination during surgery using the airseal insufflation system: video vignette. Colorectal Dis. https:// doi.org/10.1111/codi.15255

32. Khan MF, Cahill RA (2020) Carbon dioxide gas leaks during transanal minimally invasive surgery. Tech Coloproctol. https:// doi.org/10.1007/s10151-020-02284-9

33. Khan MF, Dalli J, Cahill RA (2020) Gas aerosol jetstreams from trocars during laparoscopic surgery-a video vignette. Colorectal Dis. https://doi.org/10.1111/codi.15215
34. Mintz Y, Arezzo A, Boni L, Chand M, Brodie R, Fingerhut A (2020) the Technology Committee of the European Association for Endoscopic Surgery A Low-cost, Safe, and Effective Method for Smoke Evacuation in Laparoscopic Surgery for Suspected Coronavirus Patients. Ann Surg 272(1):e7-e8. https://doi. org/10.1097/SLA.0000000000003965

Publisher's Note Springer Nature remains neutral with regard to jurisdictional claims in published maps and institutional affiliations.

\section{Affiliations}

\section{Alberto Arezzo ${ }^{1}\left(\right.$ Nader Francis $^{2,3} \cdot$ Yoav Mintz $^{4} \cdot$ Michel Adamina $^{5,6} \cdot$ Stavros A. Antoniou $^{7,8} \cdot$ Nicole Bouvy $^{9}$. Catalin Copaescu ${ }^{10}$. Nicolò de Manzini ${ }^{11}$. Nicola Di Lorenzo ${ }^{12}$. Salvador Morales-Conde ${ }^{13}$ - Beat P. Müller-Stich ${ }^{14}$. Felix Nickel ${ }^{14}$. Dorin Popa ${ }^{15}$. Diana Tait ${ }^{16}$. Cenydd Thomas ${ }^{17}$. Susan Nimmo ${ }^{18}$. Dimitrios Paraskevis ${ }^{19}$. Andrea Pietrabissa ${ }^{20}$. The EAES Group of Experts for Recovery Amid COVID-19 Pandemic}

1 Department of Surgical Sciences, University of Torino, Corso AM Dogliotti 14, 10126 Torino, Italy

2 Department of General Surgery, Yeovil District Hospital NHS Foundation Trust, Yeovil, UK

3 The Griffin Institute, The Northwick Park Institute for Medical Research, Northwick Park and St Marks Hospital, Watford Road, Harrow, Middlesex, London HA1 3UJ, UK

4 Department of Surgery, Hadassah, Hebrew University Medical Center, Jerusalem, Israel

5 Department of Surgery, Clinic of Visceral and Thoracic Surgery, Cantonal Hospital Winterthur, Winterthur, 8401 Zurich, Switzerland

6 Faculty of Medicine, University of Basel, 4051 Basel, Switzerland

7 Medical School, European University Cyprus, Nicosia, Cyprus

8 Department of Surgery, Mediterranean Hospital of Cyprus, Limassol, Cyprus

9 Department of Surgery, Maastricht University Medical Centre, Maastricht, The Netherlands

10 Department of Gastrointestinal and Bariatric Surgery, Ponderas Academic Hospital, Bucharest, Romania
11 General Surgery Clinic, Department of Medical, Surgical and Health Sciences, University of Trieste, University Hospital of Trieste, Trieste, Italy

12 Department of Surgical Sciences, University of Rome "Tor Vergata", Rome, Italy

13 Unit of Innovation in Minimally Invasive Surgery, Department of General and Digestive Surgery, University Hospital "Virgen del Rocio", University of Sevilla, Sevilla, Spain

14 Department of General, Visceral and Transplantation Surgery, Heidelberg University Hospital, Heidelberg, Germany

15 General Surgery, Linköping University Hospital, Linköping, Sweden

16 The Royal Marsden NHS Foundation Trust, London, UK

17 Department of Radiology, Yeovil District Hospital NHS Foundation Trust, Higher Kingston, Yeovil, UK

18 Department of Anaesthesia, Critical Care and Pain Medicine, Western General Hospital, Edinburgh, Scotland

19 Department of Hygiene, Epidemiology and Medical Statistics, Medical School, National and Kapodistrian University of Athens, Athens, Greece

20 Department of Surgery, Fondazione IRCCS Policlinico San Matteo, Pavia, Italy 